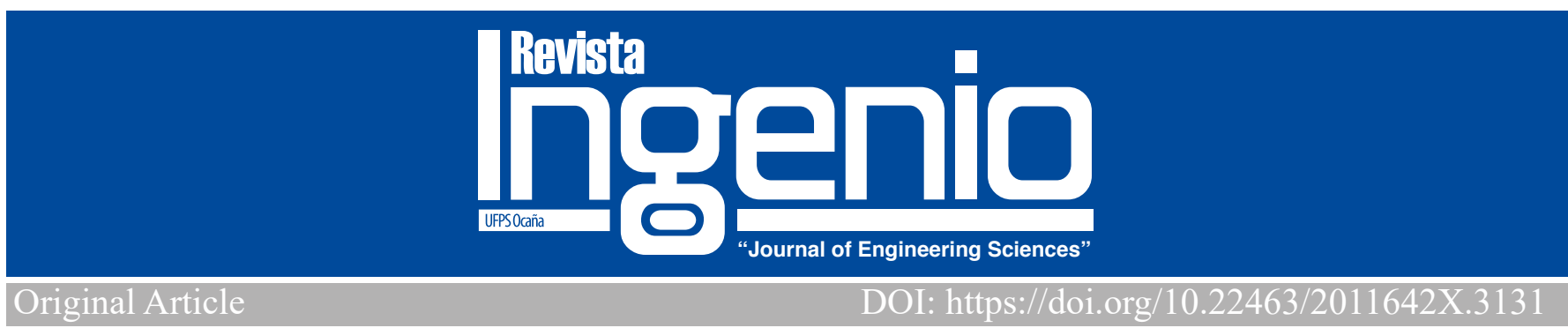

\title{
Consideraciones técnicas para la interventoría técnica de procesos constructivos de proyectos de edificaciones de concreto reforzado
}

Technical considerations for the technical supervision of construction processes of reinforced concrete building projects

\author{
Esp. Jesús David Márquez Montejo ${ }^{1}$, Esp. Elkin Yesid Barbosa Quintero², PhD. Nelson Afanador García ${ }^{3}$
}

${ }^{\prime}$ Grupo de investigación en Geotecnia y Medio Ambiente (GIGMA), Universidad Francisco de Paula Santander Ocaña, Colombia, jdmarquezm@ufpso.edu.co, Orcid: 0000-0002-8195-5583 ${ }^{2}$ Grupo de investigación en Geotecnia y Medio Ambiente (GIGMA), Universidad Francisco de Paula Santander Ocaña, Colombia, eybarbosaq@ufpso.edu.co, Orcid: 0000-0002-1091-619X ${ }^{3}$ Civil Engineering Research Group (CERG), Universidad Francisco de Paula Santander Ocaña, Colombia, nafanadorg@ufpso.edu.co, Orcid: 0000-0001-5463-2036

Cómo citar: J. D. Márquez-Montejo, E. Y. Barbosa-Quintero \& N. Afanador-García, “Consideraciones técnicas para la interventoría técnica de procesos constructivos de proyectos de edificaciones de concreto reforzado", Revista Ingenio, vol. 15(1), pp.44-50, 2018

\begin{tabular}{|c|c|}
\hline & RESUMEN \\
\hline Palabras claves: & $\begin{array}{l}\text { La Universidad Francisco de Paula Santander Ocaña (UFPSO), actualmente se expande en edificaciones de uso especial, construidas } \\
\text { con sistema estructural de pórtico de concreto reforzado, las cuales requieren de supervisión y control a los procesos de construcción. }\end{array}$ \\
\hline \multirow[t]{2}{*}{$\begin{array}{l}\text { Concreto, edificación, } \\
\text { interventoría, normativa, } \\
\text { supervisión }\end{array}$} & $\begin{array}{l}\text { Teniendo en cuenta que el municipio de Ocaña se ubica en una zona de amenaza sísmica intermedia donde las estructuras se diseñan } \\
\text { con capacidad de disipación de energía moderada (DMO); es importante conocer los criterios y controles técnicos para proyectos } \\
\text { de edificaciones que le ayuden al interventor y/o supervisor a tomar decisiones en las revisiones previas y etapas de construcción } \\
\text { de las obras. Se presenta una serie de criterios y controles técnicos establecidos en las normativas y bibliografias relacionadas a los } \\
\text { materiales de construcción y procesos constructivos de la cimentación, el sistema de pórtico y losas aligerada de concreto reforzado. } \\
\text { El resultado de la investigación fue la organización de la normatividad; consideraciones de los criterios técnicos; y la secuencia de los } \\
\text { controles técnicos aplicados a los materiales de construcción y procesos constructivos para los proyectos de edificación de la UFPSO. }\end{array}$ \\
\hline & ABSTRACT \\
\hline Key words: & $\begin{array}{l}\text { The Francisco de Paula Santander Ocaña University (UFPSO) is currently expanding into buildings for special use, built with } \\
\text { a reinforced concrete frame structural system, which require supervision and control of the construction processes. Taking into }\end{array}$ \\
\hline $\begin{array}{l}\text { Concrete, edification, } \\
\text { audit, normative, } \\
\text { supervision }\end{array}$ & $\begin{array}{l}\text { account that the municipality of Ocaña is located in an intermediate seismic hazard zone where the structures are designed with a } \\
\text { moderate energy dissipation capacity (DMO); It is important to know the criteria and technical controls for building projects that } \\
\text { help the controller and / or supervisor to make decisions in the preliminary reviews and construction stages of the works. A series } \\
\text { of criteria and technical controls established in the regulations and bibliographies related to construction materials and construction } \\
\text { processes of the foundation, the portal system and lightened reinforced concrete slabs are presented. The result of the investigation } \\
\text { was the organization of regulations; considerations of technical criteria; and the sequence of technical controls applied to construction } \\
\text { materials and construction processes for UFPSO building projects. }\end{array}$ \\
\hline
\end{tabular}

\section{Introducción}

Las edificaciones verticales son las construcciones de obras civiles que más generan impacto, dentro de la industria de la construcción, puesto que prestan un servicio comunidad con diferentes usos como el residencial, institucional, comercial y/o industrial. En Colombia han acontecido desastres como los edificios Bernavento y el Space, que han sacudido a la sociedad colombiana dejando grandes pérdidas humanas y económicas. La razón, diseños estructurales, procesos constructivos, calidad de materiales y en general aspectos técnicos, que no fueron supervisados por los organismos de control.
El colapso de la torre 6 del conjunto residencial Space en el año 2013 abrió la ventana para la creación de la ley 1796 de 2016 [1], la cual se enfoca en el incremento de la seguridad de las edificaciones y el fortalecimiento de la función pública que ejercen los curadores urbanos. Con esta ley se espera que los diseñadores estructurales y constructores respeten las normas como, el Reglamento Colombiano de Construcción Sismo Resistente (NSR-10) [2]. Además, sea conscientes con la supervisión técnica e interventoría, pues son de gran importancia en la ejecución de estas obras; ya que de ellas depende el cumplimiento de las especificaciones técnicas de un proyecto. 
La UFPSO se encuentra ubicada en el municipio de Ocaña, departamento Norte de Santander, perteneciente a zona de amenaza sísmica intermedia, según Apéndice A.4 de [2] con coeficientes de aceleración $A a=0,20 \mathrm{y} \mathrm{Av}=0,15$. Por lo anterior, las estructuras construidas se pueden diseñar con capacidad de disipación de energía moderada, y estas deben ser diseñadas y ejecutadas con criterios y procesos constructivos adecuados bajo la normatividad vigente. La presente investigación pretende dar a conocer los criterios técnicos o normativos para los materiales de construcción y procesos constructivos de obras civiles tipo edificación que actualmente se vienen ejecutando en la UFPSO.

Para determinar el alcance de la siguiente investigación, se identificó mediante el plan maestro de la UFPSO, que el sistema estructural de resistencia sísmica con mayor frecuencia de construcción es el sistema de pórticos resistentes a momento de concreto reforzado. El grupo de uso de estas edificaciones se clasifica en el grupo III de atención a la comunidad, según la sección A.2.5.1 - Grupo de Uso, del Título A [2]. El objetivo de la investigación es ofrecer a la Universidad Francisco de Paula Santander Seccional Ocaña, un conjunto de criterios y controles técnicos basados en la normatividad vigente para la interventoría y/o supervisión técnica; con enfoque a materiales de construcción y procesos constructivos del sistema estructural de pórtico y losas aligeradas de concreto reforzado que se ejecutan dentro del plan maestro.

\section{Metodología}

La investigación fue desarrollada a partir de un enfoque descriptivo cualitativo, donde se da a conocer criterios de control para los materiales de construcción y procesos constructivos de edificaciones de concreto reforzado con sistema estructural de pórtico; proyectos ejecutados en el plan maestro de la UFPSO. Para el desarrollo de la propuesta se realizaron diferentes fases, las cuales se describen a continuación:

Fase 1. Revisión documental de diferentes Normas Técnicas Colombianas (NTC), Reglamento Colombiano de Construcción Sismo Resistente NSR-10, normatividad internacional y bibliografía en general; con el fin de identificar la normatividad aplicable a los materiales de construcción y procesos constructivos del sistema estructural de pórtico y losas aligeradas de concreto reforzado de los proyectos de edificación de la UFPSO.

Fase 2. Identificación de las consideraciones técnicas para criterios técnicos y normativos enfocados a los materiales de construcción, ejecución del sistema estructural de pórtico y procesos constructivos de losas aligeradas de concreto reforzado.
Fase 3. Establecer las actividades para la verificación de controles técnicos en la interventoría de los procesos constructivos del sistema estructural de pórtico y losas aligeradas de concreto reforzado de las edificaciones de la UFPSO.

\section{Resultados}

Los resultados encontrados para cada de las fases están descritas en las siguientes secciones:

\subsection{Revisión documental}

Realizando la revisión documental de las NTC, la NSR-10, normas internacionales y bibliografía en general, se identificó la normativa aplicable a los materiales de construcción y a los procesos constructivos; enfocada al sistema de pórtico y losas aligeradas de concreto reforzado para los proyectos de edificaciones. Para los materiales de construcción se identificaron las siguientes normas:

Acero de refuerzo. Los controles técnicos en obra deben estar sujetos a la siguiente normatividad:

- Capitulo C.3.5, Titulo C, NSR-10, Acero de refuerzo [2].

- NTC 2289 (o ASTM A706M). Barras corrugadas y lisas de acero de baja aleación, para refuerzo de concreto [3].

Cemento. Los materiales cementantes empleados en la obra deben corresponder a los que se han tomado como base para la selección de la dosificación del concreto [4]. Los controles técnicos del cemento deben estar sujetos a la siguiente normatividad:

- Capítulo C.3.2, Titulo C, NSR-10, Materiales cementantes [2].

- NTC 121: Especificación de desempeño para cemento hidráulico [5].

- ASTM C150: Especificación Normalizada para Cemento Portland [6].

Agregados pétreos. Aunque los agregados se consideran materiales inertes que actúan como relleno, representan entre el $60 \%$ al $80 \%$ del volumen total del concreto. Las características de los agregados influyen considerablemente en las proporciones de la mezcla y en el costo del concreto [7].

Los controles técnicos en obra para los diferentes agregados deben estar sujetos a la siguiente normatividad:

- Capítulo C.3.3, Título C, NSR-10, Agregados [2].

- NTC 174 (ASTM C33): Concretos. Especificaciones de los agregados para concreto [8].

- NTC 129: Ingeniería Civil y Arquitectura. Práctica para la toma de muestras de agregados [9].

- NTC 3674: Ingeniería Civil y Arquitectura. Práctica 
para la reducción del tamaño de las muestras de agregados, tomadas en campo, para la realización de ensayos [10].

Agua. El propósito del agua en la mezcla de concreto es combinarla con el cemento en el proceso de hidratación, cubrir el agregado y hacer manejable la mezcla. Los controles técnicos en obra para el agua, deben estar sujetos a la siguiente normatividad:

- Capítulo C.3.4, Titulo C, NSR-10, Agua [2].

- NTC 3459 (ASTM 1602M): Concretos. Agua para la elaboración de concreto [11].

Para los procesos constructivos se identificó la siguiente normatividad:

- Título C, NSR-10. Concreto Estructural [2].

- Título H, NSR-10. Estúdios Geotécnicos [2].

- NTC 3318 (ASTM C94): Producción de concreto [12].

- NTC 396 (ASTM C143/C-143M-2015a): Concretos. Método de ensayo para determinar el asentamiento del concreto [13].

- GTC 167: Concretos. Guía acerca de la contracción del concreto [14].

- NTC 673 (ASTM C39): Concretos. Ensayo de Resistencia a compresión de especímenes cilíndricos de concreto [15].

- NTC 454 (ASTM C172): Ingeniería civil y arquitectura. Concreto fresco. Toma de muestras [16].

- NTC 550 (ASTM C31M). Concretos. Elaboración y curado de especímenes de concreto en el sitio de trabajo [17].

- NTC 2275: Ingeniería civil y arquitectura. Procedimiento recomendado para la evaluación de los resultados de los ensayos de resistencia del concreto [18].

- ACI 347: Guía para el diseño, construcción y materiales de cimbras para concreto [19].

3.2 Identificación de consideraciones técnicas para criterios técnicos y normativos.

Una vez realizada la revisión documental, se establecieron las consideraciones para los criterios técnicos y normativos de los materiales de construcción y los diferentes procesos constructivos. Para los materiales de construcción se identificaron las siguientes consideraciones:

Acero de refuerzo. Principales criterio técnicos y normativos para el control en obra:

- Realizar las verificaciones en sitio según la NTC 2289 [3]: separación de resaltes, longitud de resaltes, ángulo de resalte y peso.

- Identificar la referencia de cada barra en obra para su correcto almacenamiento.
- Almacenar el acero de refuerzo en anaqueles con cubierta y adaptados para dicho fin.

- El acero de refuerzo se debe almacenar por clasificación de longitud y diámetro.

Cemento. Principales criterio técnicos y normativos para el control en obra:

- El cemento debe ser almacenado de manera que se permita fácil acceso para la inspección eidentificación de cada despacho. El almacenamiento, ya sea bodega, contenedor, o empaque, debe garantizar la protección del cemento de la hidratación y minimizar el endurecimiento [5].

- Se debe solicitar al contratista las diferentes certificaciones, para verificar el tipo y la calidad del material cementante.

- Mantener el cemento en condiciones adecuadas de almacenamiento, en un lugar seco, bajo techo, separado de paredes y pisos, protegido de la humedad, evitando el contacto con el agua [20]. Se recomienda las siguientes separaciones: separados de muros mayor a $50 \mathrm{~cm}$; aislados del suelo mayor a 30 $\mathrm{cm}[21]$.

- Optimizar el almacenamiento del cemento, minimizando el número de sacos por pila para evitar la compactación. Recomendable almacenar en pilas de menos de 10 sacos [20].

Agregados pétreos. Principales criterio técnicos y normativos para el control en obra:

- Se permite el uso de agregados que han demostrado a través de ensayos, o por experiencias prácticas que producen concreto de resistencia y durabilidad adecuadas, siempre y cuando sean aprobados por el Supervisor Técnico Independiente [22].

- El tamaño máximo nominal del agregado grueso no debe ser superior a: (a) 1/5 de la menor separación entre los lados del encofrado, ni a (b) $1 / 3$ de la altura de la losa, ni a (c) 3/4 del espaciamiento mínimo libre entre las barras o alambres individuales de refuerzo, paquetes de barras, tendones individuales, paquetes de tendones o ductos [22].

Estas limitaciones se pueden omitir si a juicio del profesional facultado para diseñar la trabajabilidad y los métodos de compactación son tales que el concreto se puede colocar sin la formación de hormigueros, vacíos o segregación en la mezcla [22].

- Se debe solicitar al contratista los ensayos para materiales pétreos: Granulometría (NTC 174), masa unitaria, densidad, porosidad y absorción, textura, forma y pasa tamiz 200, para verificar que los parámetros de los materiales son equivalentes a los utilizados en el diseño de mezcla. 
- El almacenamiento y manejo de los agregados pétreos debe hacerse de tal manera que no altere su composición y granulometría. Es decir, sobre pisos adecuados y separados por lotes, remisiones o viajes para evitar que se mezclen entre sí los agregados de diferente clasificación. No usar aquellos agregados que por cualquier circunstancia se hayan contaminado [23].

Agua. Principales criterio técnicos y normativos para el control en obra:

- El agua para la mezcla debe estar libre de materias orgánicas, álcalis, ácidos y aceites. Por lo general, el agua potable también sirve para mezclarla con cemento [7].

- Se deberá también hacer pruebas para verificar que el tiempo del fraguado del cemento no sea afectado por impurezas en el agua de mezcla [7].

- Controlar principalmente el contenido de azúcares disueltas y materia orgánica. Además, identificar concentraciones altas de cloruros y sulfatos.

- Controlar el PH, orden de magnitud recomendado entre 6 y 8.

- Diseñar por durabilidad según el capítulo C.4 del título C de la NSR-10 [2].

En la figura 1, se puede observar los procesos constructivos contemplados en la investigación, para la identificación de los criterios técnicos y normativos.

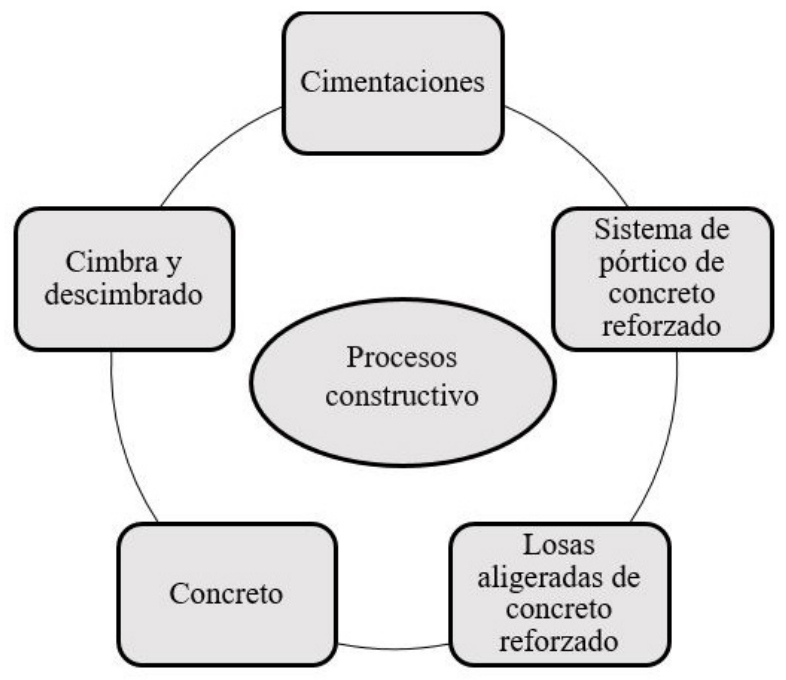

Figura 1. Procesos constructivos generales

Cimentaciones. El tipo de cimentación utilizada dentro del Plan Maestro de la Universidad Francisco de Paula Santander seccional Ocaña para la construcción de edificaciones, consiste en un sistema superficial conformado por zapatas aisladas, pedestales y vigas de cimentación en concreto reforzado.
A continuación, se describen las consideraciones de los principales criterios identificados para las actividades de la cimentación:

- Revisión en los planos estructurales de las dimensiones de las zapatas, pedestales y vigas de cimentación.

- Se debe realizar la secuencia de excavaciones según la sección H.8.2.4 del título H de la NSR-10 [2].

- De ser necesario, las excavaciones se realizarán por etapas según lo descrito en la sección H.8.2.4, del título H de la NSR-10 [2].

- Se debe contar con un plan de contingencia para excavaciones según lo contemplado en la sección H.8.2.6 del título H de la NSR-10 [2].

- Las distancias mínimas para acopio de material sobrante de excavación deben ser mayores a $0.60 \mathrm{~m}$ [24].

- Debe mantenerse una distancia segura entre los trabajadores, recomendable mínimo dos (2) metros [24].

- La excavación en las partes inferiores de los taludes está prohibida, debido al gran riesgo de desmoronamiento [24].

- Protección de concreto para el refuerzo; concreto colocado contra el suelo y expuesto permanentemente a él, debe tener un recubrimiento mínimo de $75 \mathrm{~mm}$ [25].

Sistema de pórtico de concreto reforzado. Los criterios para la construcción de los elementos estructurales de pórticos de concreto reforzado, como vigas y columnas se describen a continuación, con el fin de brindar parámetros de referencia.

- Verificación en los planos estructurales de las dimensiones de vigas y columnas.

- Se debe revisar en los planos estructurales los diferentes despieces del acero de refuerzo con el fin de garantizar parámetros como: separación de estribos, recubrimientos, cuantías de acero de refuerzo longitudinal, ubicación y longitud de traslapos, longitud de ganchos, diámetros mínimos de doblado, entre otros.

- En el momento que es colocado el concreto, el refuerzo debe estar libre de barro, aceite u otros recubrimientos no metálicos que reduzcan la adherencia [26].

- El refuerzo, excepto el acero de preesforzado, con óxido, escamas o una combinación de ambos, debe considerarse satisfactorio si las dimensiones mínimas (incluyendo la altura de los resaltes del corrugado) y el peso de una muestra limpiada utilizando un cepillo de alambre de acero, cumple con las especificaciones NTC (o ASTM en su defecto) aplicables indicadas en C.3.5 [26].

Losas aligeradas de concreto reforzado. Los criterios 
principales para la construcción de losas aligeradas de concreto reforzado se describen a continuación:

- Revisión en los planos estructurales de las dimensiones de la losa.

- Verificar en los planos estructurales la distribución del acero longitudinal y transversal.

- Garantizar la protección de concreto para el refuerzo según los planos estructurales (para losas con barras $\mathrm{N}^{\circ} 11$ o menores debe ser mínimo de $20 \mathrm{~mm}$ [25]).

Concreto. Mezcla de cemento Pórtland o cualquier otro cemento hidráulico, agregado fino, agregado grueso y agua, con o sin aditivos. Los principales criterios para obtener un concreto de calidad son los siguientes:

- Verificar las tolerancias en el asentamiento de la mezcla según lo dispuesto por la NTC 3318 [12].

- Garantizar la dosificación del concreto para alcanzar la resistencia a la compresión especificada y satisfacer los criterios de durabilidad del capítulo C. 4 del título C de la NSR-10 [2].

- Los requisitos para f'c deben basarse en ensayos de cilindros, hechos y ensayados como se establece en C.5.6.3 [27].

- A menos que se especifique lo contrario f'c debe basarse en ensayos a los 28 días. Si el ensayo no es a los 28 días, la edad de ensayo para obtener f'c debe indicarse en los planos o especificaciones de diseño [27].

- El concreto debe ensayarse de acuerdo a los requisitos de la sección C.5.6.2 a C.5.6.5, del título C de la NSR10 [2].

- Las muestras para ensayos de resistencia deben tomarse de acuerdo con NTC 454 (AST C172) [27].

- Los cilindros para los ensayos de resistencia deben ser fabricados y curados en laboratorio de acuerdo con NTC 550 (ASTM C31M), y deben ensayarse de acuerdo con NTC 673 (ASTM C39M). Los cilindros deben ser de 100 por $200 \mathrm{~mm}$ o de 150 por $300 \mathrm{~mm}$ [27].

- La preparación del equipo y lugar de colocación del concreto deben cumplir lo especificado en la sección C.5.7.1 del título C de la NSR-10 [2].

- El mezclado del concreto debe cumplir los requisitos de la sección C.5.8 del título C de la NSR-10 [2].

- El transporte de la mezcla de concreto debe cumplir los requisitos de la sección C.5.9 del título $\mathrm{C}$ de la NSR-10 [2].

- La colocación de la mezcla de concreto debe cumplir los requisitos de la sección C.5.10 del título $\mathrm{C}$ de la NSR-10 [2].

- El curado del concreto debe satisfacer los requisitos de la sección C.5.11 del título C de la NSR-10 [2].

- Las juntas de construcción deben cumplir los requisitos de la sección C.6.4 del título C de la NSR-10 [2].
Cimbra y descimbrado. Cimbra, es un sistema total de soporte para el concreto fresco, incluyendo los moldes o forros que se colocan en contacto con el concreto, así como todos los elementos de soporte, accesorios y contraventeos necesarios [19].

A continuación, se describen los principales criterios para la actividad de cimbra y descimbrado:

- El diseño y construcción de la cimbra, deben ser responsabilidad del contratista [19].

- Las cimbras y encofrados deben ser esencialmente y suficientemente herméticos para impedir la fuga del mortero [28].

- Las cimbras y encofrados deben estar adecuadamente arriostrados o amarrados entre sí, de tal manera que conserven su posición y forma [28].

- Tener una provisión extra de puntales, materiales y equipos que pudiera necesitarse en caso de emergencia [19].

- Las cimbras deben limpiarse perfectamente de toda basura, mortero, materiales extraños y revestirse con un agente desmoldante antes de cada uso [19].

- Los revestimientos de las cimbras deben aplicarse antes de colocar el acero de refuerzo y no deben usarse en cantidades que lleguen a las varillas o a las juntas de construcción del concreto [19].

- No se recomienda el apuntalamiento con puntales apilados, es decir un solo poste formado por dos o más puntales uno sobre otro porque se le considera una práctica peligrosa [19].

- La cimbra debe retirarse de tal manera que no se afecte negativamente la seguridad o funcionamiento de la estructura. El concreto expuesto por el descimbrado debe tener suficiente resistencia para no ser dañado por las operaciones de descimbrado [28].

- El cimbrado lateral de las losas y vigas deben tener un refuerzo con una distancia de apoyo mínima de 30 $\mathrm{cm}$.

- Los refuerzos laterales de la cimbra de losas y vigas deben estar ubicados de tal manera que guarden el espacio suficiente para que el personal de obra pueda desplazarse alrededor de la misma.

- Con anterioridad al inicio de la construcción, el constructor debe definir un procedimiento y una programación para la remoción de los apuntalamientos y para la instalación de los apuntalamientos, y para calcular las cargas transferidas a la estructura durante el proceso [28].

\subsection{Estructura para el control técnico}

La estructura para el control técnico, se basa en el control de ejecución prescrita en la sección I.4.3.7, Título I, NSR-10 [2], ver figura 2. 


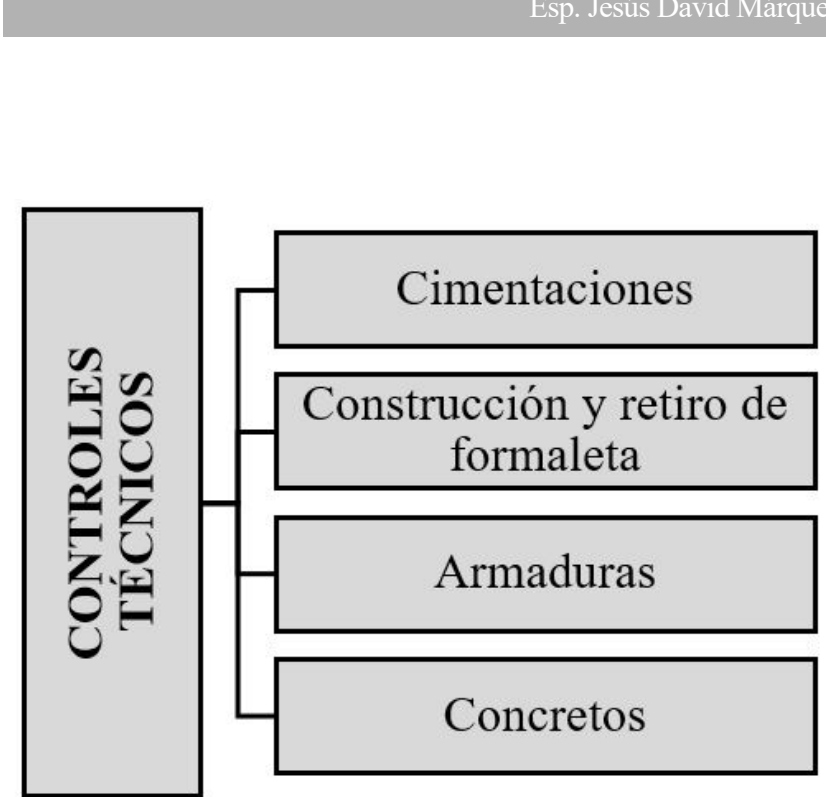

Figura 2. Control técnico

En los controles técnicos se disponen los puntos mínimos que el supervisor técnico debe inspeccionar. Estos controles se enfocan solo en la construcción del sistema estructural de pórtico y losa aligerada de concreto reforzado. A continuación, se describen las actividades principales a las que se deben dar seguimiento en función de los controles técnicos principales.

Cimentaciones. Se debe dar seguimiento al replanteo geométrico, dimensiones de excavaciones, limpieza fondo de excavaciones, sistema de drenaje, estratos y niveles de fundación y protección de excavaciones.

Construcción y retiro de formaletas. Se debe dar seguimiento al alineamiento de formaletas; acabado de superficies y su verticalidad; resistencia y estabilidad ante asentamientos; aprobación cálculo de cimbras; limpieza e impermeabilidad; aberturas de inspección; y descimbrado.

Armaduras. Se debe dar seguimiento al grado del acero (fy), diámetro, numero de barras, gancho y longitud; empalmes; colocación de recubrimientos, distancia entre barras, sujeción; y limpieza de barras y de la zona de vaciado $\mathrm{y}$ aspecto superficial.

Concretos. Se debe dar seguimiento a actividades como: aprobación de los diseños de mezcla; medios y procedimientos de mezclado; medios y procedimientos del transporte; medios y procedimientos de colocación y compactación; medidas y procedimientos para la toma de muestras; tiempo transcurrido entre mezcla y colocación; homogeneidad y consistencia de los concretos; provisiones para vaciado; definición de juntas de construcción; preparación de superficies, juntas de construcción y dilatación; y sistemas y procedimientos de curado.

\section{Conclusiones}

A la hora de aplicar los diferentes criterios normativos, es importante conocer e informarse de la vigencia de la norma consultada para no implementar criterios que puedan ser anulados o modificados. En la revisión documental, se encontró que para los materiales de construcción y procesos constructivos las normas aplicables fueron las diferentes Normas Técnicas Colombianas (NTC); algunas normas internacionales como American Society for Testing and Materials (ASTM) y el American Concrete Institute (ACI); y principalmente el Reglamento Colombiano de Construcción Sismo Resistente NSR-10, las cuales fueron complementadas con manuales técnicos de construcción y bibliografia variable.

Referente a las consideraciones técnicas es de gran importancia conocer las medidas que se pueden tomar para la seguridad de los procedimientos que se deben aplicar en el sitio de la obra; permitiendo al interventor desarrollar su trabajo de una manera más eficiente. Conocer las consideraciones técnicas de criterios técnicos y normativos, permiten un rápido seguimiento a los materiales de obra y los procesos constructivos, manteniendo la calidad de las edificaciones y evitando errores que afecten de forma negativa el cronograma de los proyectos.

La identificación de los controles técnicos según el Título I de la NSR-10 para el sistema de pórtico y losas aligeradas en las edificaciones de concreto reforzado, establece un procedimiento de inspección de las diferentes actividades a desarrollar; ventaja para el interventor y/o supervisor que al combinarlo con los criterios técnicos y normativos determina, en la ejecución de las actividades, si la normativa vigente se está aplicando de forma correcta.

\section{Referencias}

[1] Ley 1796, Ministerio de vivienda, ciudad y territorio, Bogotá, 13 de julio 2016.

[2] Asociación Colombiana de Ingeniería Sísmica, Reglamento Colombiano de Construcción Sismo Resistente NSR-10, Bogotá, 2010.

[3] Instituto Colombiano de Normas Técnicas y Certificación (ICONTEC), Barras corrugadas y lisas de acero de baja aleación, para refuerzo de concreto, NTC 2289, Bogotá, 2007.

[4] Asociación Colombiana De Ingeniería Sísmica, "Titulo C: capitulo C.3.2 - Materiales cementantes", Reglamento Colombiano de Construcción Sismo Resistente NSR-10, BOGOTÁ: AIS, 2010, pp. C-42.

[5] Instituto Colombiano de Normas Técnicas y Certificación (ICONTEC), Especificación de desempeño para cemento hidráulico, NTC 121, 
Bogotá, 2014.

[6] American Society for Testing and Materials (ASTM), Standard Specification for Portland Cement, ASTM C150M, United State of America, 2017.

[7] T. W. Love, El concreto en la construcción, México: Trillas, 2006.

[8] Instituto Colombiano de Normas Técnicas y Certificación (ICONTEC), Concretos. Especificaciones de los agregados para el concreto, NTC 174, Bogotá, 2000.

[9] Instituto Colombiano de Normas Técnicas y Certificación (ICONTEC), Práctica para la toma de muestras de agregados, NTC 129, Bogotá, 1995.

[10] Instituto Colombiano de Normas Técnicas y Certificación (ICONTEC), Ingeniería civil y arquitectura. Práctica para la reducción del tamaño de las muestras de agregados, tomadas en campo, para la realización de ensayos, NTC 3674, Bogotá, 1995.

[11] Instituto Colombiano de Normas Técnicas y Certificación (ICONTEC), Concretos. Agua para la elaboración de concreto, NTC 3459, Bogotá, 2001.

[12] Instituto Colombiano de Normas Técnicas y Certificación (ICONTEC), Producción de concreto, NTC 3318, Bogotá, 2008.

[13] Instituto Colombiano de Normas Técnicas y Certificación (ICONTEC), Concretos. Método de ensayo para determinar el asentamiento del concreto, NTC 396, Bogotá, 1992.

[14] Instituto Colombiano de Normas Técnicas y Certificación (ICONTEC), Concretos. Guía acerca de la contracción del concreto, GTC 167, Bogotá, 2007.

[15] Instituto Colombiano de Normas Técnicas y Certificación (ICONTEC), Concretos. Ensayo de resistencia a compresión de especímenes cilíndricos de concreto, NTC 673, Bogotá, 2010.

[16] Instituto Colombiano de Normas Técnicas y Certificación (ICONTEC), Ingeniería civil y arquitectura. Concreto fresco. Toma de muestras, NTC 454, Bogotá, 2011.

[17] Instituto Colombiano de Normas Técnicas y Certificación (ICONTEC), Concretos. Elaboración y curado de especímenes de concreto en el sitio de trabajo, NTC 550, Bogotá, 2017.

[18] Instituto Colombiano de Normas Técnicas y Certificación (ICONTEC), Ingeniería civil y arquitectura. Procedimiento recomendado para la evaluación de los resultados de los ensayos, NTC 2275, Bogotá, 1997.

[19] The American Concrete Institute (ACI), Guía para el diseño, construcción y materiales de cimbras para concreto, ACI 347-04, United State of America, 2009.

[20] Argos, Cemento gris de uso general (Versión 1), 2013. [En línea]. Available: https://www.argos.co/Media/
Panama/images/01\%20CEMENTO\%20USO $\% 20$ GENERA.pdf.

[21] M. Mena, Presentación de clase de especialización en interventoría de obras civiles: Concretos, Ocaña, 2017.

[22] Asociación Colombiana de Ingeniería Sísmica, "Título C: capitulo C.3.3 - Agregados", Reglamento Colombiano de Construcción Sismo Resistente NSR10, Bogotá, 2010.

[23] J. Rivero, Manual técnico de construcción, 2008. [En línea]. Available: https://usuaris.tinet.cat/oriolcid/ manualconstruccion.pdf.

[24] Ministerio del trabajo, Guía trabajo seguro en excavaciones, 2014. [En línea]. Available: https:// www.mintrabajo.gov.co/documents/20147/51963/ $\mathrm{Gu} \% \mathrm{C} 3 \% \mathrm{ADa}+\mathrm{de}+\mathrm{Escavaciones}+09+\mathrm{FEB}$. pdf/1892a703-82bc-3652-cdd7-5380e6e2079c.

[25] Asociación Colombiana de Ingeniería Sísmica, "Titulo C: capitulo C.7.7 - Protección de concreto para el refuerzo", Reglamento Colombiano de Construcción Sismo Resistente NSR-10, Bogotá, 2010.

[26] Asociación Colombiana de Ingeniería Sísmica, "Título C: capitulo C.7.4 - Condiciones de la superficie del refuerzo", Reglamento Colombiano de Construcción Sismo Resistente NSR-10, Bogotá, 2010.

[27] Asociación Colombiana de Ingeniería Sísmica, "Titulo C: capitulo C.5 - Calidad del concreto, mezclado y colocación", Reglamento Colombiano de Construcción Sismo Resistente NSR-10, Bogotá, 2010.

[28] Asociación Colombiana De Ingeniería Sísmica, "Título C: capítulo C.6 - Cimbras y encofrados, embebidos y juntas de construcción", Reglamento Colombiano de Construcción Sismo Resistente NSR10, Bogotá, 2010. 